\title{
ACERCA DE LO OBSERVABLE Y LO TEÓRICO EN PSICOANÁLISIS
}

El psicoanálisis ha sido una de las disciplinas más discutidas dentro de la problemática intelectual de este siglo. Desde que Freud lo forjó ha sido objeto de innumerables críticas provenientes tanto de las áreas médicas y biológicas como de las humanísticas. Ha sido pretendidamente desterrado de la psiquiatría con sus enfoques organicistas, de la psicología a través del conductismo, de la sociología y la antropología a través de sus diversos esquemas conceptuales empíricos y desacreditado por ciertos enfoques filosóficos ideológicos y epistemológicos.

Una de las críticas más serias dirigidas a la teoría psicoanalítica proviene de la filosofía de la ciencia, especialmente del enfoque derivado del círculo de Viena y desarrollado hasta la actualidad por Hempel, Bunge, Popper y Nagel, entre otros. De manera general este enfoque ha cuestionado la cientificidad de la teoría psicoanalítica aduciendo la vaguedad e imprecisión de sus conceptos, el carácter ad hoc e incontrastable de sus hipótesis, la gratuidad de sus leyes, la flexibilidad de sus explicaciones, su incapacidad predictiva y su carencia metodológica: la ausencia de grupos control de experimentación para fundamentar sus postulados. Postura que ha llevado a algunos, como Bunge, a comparar al psicoanálisis con la astrología y a relegarlo al terreno de la pseudo ciencia.

Uno de los problemas fundamentales que subyace en las diversas argumentaciones epistemológicas contra el psicoanálisis es el de la oscuridad que rodea a las relaciones entre los conceptos teóricos:y los observacionales de la teoría psicoanalítica. En la medida en que se carezca de una noción más clara de estas relaciones siempre surgirán todo tipo de objeciones que girarán en torno a la fuerza lógica de las hipótesis y leyes del psicoanálisis, así como al contenido empírico de las mismas.

El problema de la relación entre el nivel teórico y el observacional en la teoría psicoanalítica incide en una encrucijada de problemas que han sido abordados desde varios puntos de vista:

a) Un enfoque que llamaré "formalista" que pretende establecer y definir un lenguaje observacional, un lenguaje teórico y las reglas de correspondencia entre ambos, reglas que constituyen un criterio de admisibilidad y de significado de los conceptos teóricos incorporados en toda teoría científica; postura ejemplificada por Carnap en su artículo "The methodological character of theoretical concepts" [1]. 
b) Un enfoque que llamaré "estructural" que corresponde al programa de reconstrucción estructural de las teorías empíricas y que explica las relaciones entre-los conceptos teóricos ${ }^{*}$ los observacionales en términos de su función o, su manejo relativo a una teoría específica, siendo teóricos aquellos que pertenécen al núcleo central de la teoría y que presuponen la aceptación de la misma, y siendo no teóricos áquellos conceptos empleados en teorías previas independientes de áquélla én cuestión y que constituye el dominio de sus aplicaciones empíricas y de base confirmatoria, tâl como lo expone el profr. Moulines [2] y [3].

c) Un enfoque que llamaré "semántico" que aborda la cúestión a través del problema de la reláción entre la mente y el cuerpo y que incluye diversos análisis dé los conceptos mentales explicándolos como "disposicionales", "variable e intercurrentes", "lenguaje privado", etc. "cuya mención de sus ramificaciones y variedades excede los límites de este trabajo y que ha sido desarrollada por una serie de investigadores 'que va desde Reyle y Wittgenstein hasta Davidson y Krypke.

"Ante tal gamà de posibilidades ôptaré pör abordar directamente fa cuestión a través del examen de un càso concreto de aplicación de los conceptos téóricos a una situación clinica particular en la que se hallan presentes todos los elementos observacionales de manera que podámos descubrir la estructura de la relación entre lo teóricô y lo observable en la teoría psicoanalíticá.

Ya en un trabajo anterior [4] me propuse diseñar un bosquejo de" análisis de las relaciones entre los conceptos teóricos del psicoanálisis y la "experiencia a través de una aproximación "formalista" en la que utilicé la distinción de Frege entre "propiedäd" y "notä" de un concepto y las rélaciones de "inclusión" y "subordinación" äplicadas respectivámente para conectar los diferentes nivelés de abstracción de las hipótesis y leyes de la teóría con el matêrial empírico. Sin embargo, creo más conveniente ahora proceder inversamente y partir de un ejemplo concreto pära descubrir "las relaciones "más "reales" o "funcionales" que de" hecho se dan" entre los" conceptos teóricos y' los observablès, relaciónes más fáciles de disecar en aplicaciones de la teoría a situaciones párticulares. 'Nó tomaré como marco de referencia ninguna aproximación semántica especifica, más bien procuraré sẹnalar los diversos problemas que vayan surgiendo en el análisis. Desde luego no pretendo partir de un punto de vista ingenuo libre de todo compromiso conceptual, sólo trato de abordar la cuestión sin un esquema preconcebido." "

El imaterial clínico que presentaré a continuación es necesariamente fragmentario, primera limitáción seria de este trabajo, ya qué la aplicación correcta dè los conceptos teóricos del psicoanálisis requiere de la totalidad de elementos clínicos y dinámicos "qué se dan en la situación analítica en 
un lapso de tiempo extenso, o como se dice en la jerga psiquiátrica, en una visión transversal y longitudinal de un paciente; sin embargo mi propósito no es agotar el examen de las relaciones entre el nivel teórico y el observable de la teoría psicoanalítica, sino sólo indicar la manera en que dicha labor puede llevarse a cabo. El material procede de un caso clínico tratado exhaustivamente por el Dr. Sérgio Mendizábal, quien amablemente me brindó su autorización ṕara aprovechar su experiencia en este ensayo. Se trata de un paciente de 17 años de sexo masculino que actualmente se encuentra internado en una institución psiquiátrica donde recibe tratamiento farmacológico y' psicoterapéutico y donde es posible controlar y observar su evolución, situación que permite la obtención de material confirmatorio de las diversas hipótesis psicoanalíticas que sobre su caso se formulen.

Los síntomas principales que el paciente presenta-son:

1) Homosexualidad pasiva con transvestismo.

2) Dos intentos de suicidio:

3) Cuadro depresivo crónico con tendencias paranoides.

A continuación presentaré un breve resumen de su historia personal y familiar que permita la construcción de algunos conceptos teóricos dé diverso nivel de abstracción que conectan el material observable de su sintomatologia actual con los elementos 'de su historia individual a través de hipótesis y leyes articuladas en modelos explicativos. Para analizar las relaciones lógicas que se dan entre los conceptos teóricos y el material observable será necesarió confeccionar algunos modelos explicativos fragmentarios de la psicodinamia del paciente que muestren con más claridad la operatividad conceptual de la teoría psicoanalítica. Familia compuesta por el padre, la madre, nuevé hermanos 'y tres medias hermanas. Clase socioeconómicà media baja.

Datos relevantes del padre. Fallecido en mayo de 1976 a los 53 años. Alcohólico crónico y farmacodependiente (benzedrinas) convivió poco con la familia por razones de trabajo. Las pocas ocasiones que permanecía en su hogar 'golpeaba brutalmente 'a los varones; yi en "especial, se ensañaba con el paciente. Fue percibido como una figura ausente y agresiva.

Datos relevantes de la mädre. De 43 años de edad; dio a luz a 4 varones antes de nacer el paciente. Deseosa de tener una niña, estimuló las actitudes femeninas del paciente (véstirse de niña $y$ jugar a las muñecas con sus hermanitas), y lo defendió de las agresiones del padre. Cuando.ella se ausentaba llevaba al paciente a vivir con sù abuela en cúya casa no había ninguna figura masculina. Se quejaba con sus hijos:de la agresividad del padre y adoptaba el papel pasivo de "la mujer sufrida":

Datos relevantes de los hermanos. El mayor, de 24 años, intentó suicidarse sin éxito, es amigo del paciente. El siguiente, de $2 x$ años, es farmacodependiente, ha sido internado en una institución psiquiátrica por presentar cuadro psicótico agudo y ha sido encarcelado por actos delictivos. El tercero 
y cuarto hermanos son farmacodependientes. A excepción del primer hermano, todos rechazaron y agredieron al paciente por sus actitudes afeminadas. Las hermanas jugaban con él a las muñecas, lo que complacía tanto al paciente como a su madre:

Datos relevantes de su historia personal. Fue intervenido quirúrgicamente a los 6 años por falta de descenso de los "testículos. Vivió grandes' temporadas de su infancia con la abuela. En la primaria se relacionaba con "las niñas y evitaba a los compañeros, quienes se mofaban de sus actitudes femeninas. Desde pequeño se vestía de mujer con las ropas de su madre y sus hermanas. Jugaba a las muñecas con ellas. Evitaba jugar con sus hermanos para evitar ser golpeado por ellos. Al percatarse el padre de las actitudes femeninas del paciente, incrementó sus agresiones al grado de amenazarlo con la muerte si se volvía homosexual. A los 8 años tuvo su primera experiencia homosexual pasiva con un compañero tres años mayor que él, ya que desde esa edad sentía atracción sexual hacia los hombres, según lo refiere él mismo.

Al enterárse el padre, le propinó una golpiza brutal, $\times$ y al regresar al día siguiente de la escuela, el paciente hizo su primer intento suicida al arrojarse desde un puente, sin embargo, sólo sufrió heridas de moderada intensidad, que fueron atendidas en un hospital de traumatología.

Desde esa fecha ha padecido episodios ć́clicos depresivos intensos, a de cir del paciente; porque su padre jamás lo aceptó. A los 15 años ha vivido fuiera-de su casa en una većindad cercana a lä cása de su abuela. Hasta los 16 años, su actividad homosexual pasiva fue esporádica: A partir de esá fecha, frẹcuentó cabarets donde actuaba papeles femeninos usando vestimenta femenina. Ahí conseguia clientes homosexuales a quienes embriagaba y estafaba: Actualmente trabaja como "secretario" de una artista; ocupándose del vestuario, maquillaje y aseo de la casa de ésta.

Breve resumen del padecimiento actual. A raiz de realizar un trabajo excesivo el paciente presentó un cuadro depresivo (insomnio, ansiedad, pérdida de apetito, tristeza, sentimientos de minusvalía) que se inició 4 meses antes de su ingreso al hospital. En estas condiciones, el it de mayo de 1977 , fecha cercana al aniversario de la muerte de su padre, el paciente incrementa su sintomatología e intenta su segundo acto suicida, el cual fue impedido por la dueña de la casa en donde habitaba y a quien el paciente llamaba "abuela". A consecuencia de esto, el paciente decide acudir al hospital donde pide le sean extirpados sus genitales y: reclama ser intervenido quirúrgicamente para ser convertido en 'mujer. Dentro de lạ sala de internamento, el paciente mostró grạn desconfianza en sus relacionè interpersonales con los demás pacientés; quejándose de sentirse observado y criticado por todos.

Tal como se sostiene actualmente, la aplicación de un segmento de una teoría para explicar un fénómeno particular implica la asunción de toda la 
teoría, la red conceptual que indirectamente se compromete en la explicitación de alguno de sus fragmentos. La explicación psicoanalítica que voy a presentar además de ser incompleta, está sustentada en la totalidad de la teoría, cuyas ramificaciones y modelos permanecerán implícitos, ya que para mi propósito; sólo basta mostrar un pequeño sector de la misma para abordar el problema de lo teórico y su relación con lo observable.

Voy a intentar explicar la conducta homosexual del paciente, su, depresión con sus intentos suicidas y sus razgos paranoides a partir de su historia personal y familiar utilizando articulaciones de conceptos psicoanalíticos de diverso nivel de abstracción que reflejen la relación entre lo teórico y lo observable.

Comenzaré con explicaciones superficiales y del sentido común que emplean términos del lenguaje cotidiano como punto de partida para llegar a las explicaciones más técnicas y abstractas.

A primera vista, el paciente siente un gran rechazo hacia la identidad masculina, no desea ser hombre a pesar de haber nacido tal. Prefiere ser mujer e incluso desea ser transformado quirúrgicamente en una persona del sexo femenino. Superficialmente esto se explica por la actitud de su madre y hermanas de estímulo de sus actitudes femeninas (jugar con muñecas y vestirse de mujer) desde su infancia,: unidos a la ausencia de un padre que estimule la identificación masculina y cuya presencia se manifiesta en actitudes de cariño hacia las mujeres y agresividad hacia los hombres, especialmente hacia el paciente. Éste sintió que la asunción de una identificación femenina era la única solución viable para garantizarse la aceptación de la madre así como el cariño de un padre que mostraba su ternura hacia las mujeres de su familia.

La explicación anterior presupone una necesidad extrema de cariño en el paciente, una gran carencia afectiva que buscaba llenar a través de su homosexualidad buscando recibir ese cariño de los hombres, y originalmente, de su padre. El paciente deseaba ser querido por su padre. Al mismo tiempo, sentía una gran insatisfacción ante el cariño de las mujeres (madre y hermanas). Aquí surge la primèra paradoja, ¿por qué buscaba el cariño del padre si éste fue brutalmente agresivo y rechazante:con él? ¿ ¿Por qué no gozó y aceptó el cariño de su madre y hermanas que sí to aceptaron? ¿Cómo se explica la orientación homosexual de su carácter ante unas condiciones familiares que parecían favorecer una orientación heterosexual?

Profundizando un poco más, se infiere que la gran insatisfacción que sentía el paciente ante el cariño de su madre y que lo hizo optar por la homosexualidad, oculta una agresión y un temor hacia la figura femenina superior aún al temor y agresión hacia su paủre. Dicha agresión y temor hacia su madre se originaron debido a la situación de abandono y deprivación afectiva que el paciente padeció en sus primeros años de vida, de ahí su gran necesidad de cariño. Para explicar estos fenómenos será necesario introducir ex- 
plicaciones más abstractas que incorporen términos teóricos y modèlos fragmentarios de la teoría psicoanalítica sobre la homosexualidad. ti is

El concepto central de ila teoría empleado para explicar la homosexualidad es el de "fijación pregenital de la libido y la agresión "en la faseroral (Freud) o en la fase simbiótica (Mahler), del desarrollo psicológico del individuo".

El Dr. Charles 'W. Socarides [5] resume admirablemente la explicáción de la homosexualidad a través del empleo de este concepto de la manera siguiente: La homosexualidad que padece el paciente pertenece al" tipo " preedípico" porque se origina en las primeras fáses del desarrollo psicosexual, anteriores al "complejo de Edipo". Este tipo de 'homosexualidad "... . se debe a una fijación a una etapa preedípica del desarrollo (del nacimiento a la edad de 3 años). Se encuentra motivada inconscientemente y surge de la ansiedad. Debido a que lärabstinencia de la práctica homosexual ocasiona angustia intolerable y debido a que la pareja debe ser. del mismö sexó, debe ser considerada una homosexualidad obligatoria. Este patrón sexual es inflexible y estereotipado Está presente una alteración severa de lá identidad sexual: en el hombre, una débil y fallidaridentificación masculina.:.: La alteración de la identidad ssexual se debe a la persistencialde la identificación femenina primaria, como resultado de la incapacidad para alcanzar la fase de separación individuación (del nacimiento a los i8-36 meses) y para desarrollar, una identidad separada e e independiente de la madrè Resulta esencial diferenciar entre una identificación femenina primaria y una secundaria. Al nacèr el niño, la unidad biológica con la madre se sustituye por ùna identificación primitiva con ella. El niño debe proseguir desde la seguridad de la identificación y la unidad con la madre hasta la sepáración activa y competenté; en el niño; a través de aproximaciones activas masculinas (fálicas)... sì esta tarea se presenta excesivamente difícily surgen defénsás patológicas, especialmente una agresividad superlativa. Estos desarrollos son de la mayor importancia para la solución de los conflictos que aparecen en la fase edípica y en la vida posterior En la fase edípica del värón; bajo la presión de la angustia de castración, aparece un tipo àdicional de identificación secundaria con la madre, que toman la forma de deseos femeninos pasivos hacia el padre. Sin embargo, por debajo de esta posición femenina en relación al padre a menudo se encuentra encubierta la relación pasiva original cón la madre: una identificación femenina primaria preedípica activa . . las ansiedades que acosan a las personas de este tipo son de una naturaleza insistente e intratable, $y$,dan lugar a una búsqueda atropellante $y$ casi continua de parejas sexuales.' La persistencia de mècànismos mentales arcaicos y primitivos ocasionan una grản cántidad de ansiedades de incorporación y proyección. La ansiedad que se,desarrólla se debe a temores de engolfamiento, disolución del ego, pérdida de la identidad y de sus límites. El acto homosexual resulta 
necesario para asegurar la supervivencia del ego y para estabilizar transitoriamente el sentido de identidad. Consecuentemente, el acto debe ser repetido frecuentemente y fuera de la necesidad interna de protegerse de los miedos paranoides ${ }_{i} \mathrm{e}$ incorporativos... El síntoma homosexual resulta egosintónico, como sus conflictos nucleares: miedo de engolfamiento, pérdida de los límites del ego, pérdida de la identidad han sufrido una transformación y se disfrazan a través del mecanismo del compromiso represivo, se hace posible la permanencia en la conciencia de la mayor parte aceptable posible de la sexualidad infantil... Hay un predominio de las características pregenitales del ego; el recuerdo se sustituye por el "actuar fuera". La finalidad del acto homosexual consiste en: la supervivencia del ego y en una reconstitución del sentido de la identidad sexual en concordancia con la anatomía. El varón adquiere la "masculinidad" a través de la identificación con su pareja sexual masculina; reasegurándose en contra de y disminuyendo el miedo de la cástración..." (pp. 297-298). A toda la explicación anterior hay que agregar que la fijación pregenital simbiótica implica la permanencia de procesos de pensamiento mágico omnipotente consistentes en la sensación de que las fantasías se realizan con el solo hecho de pensarlas con todas sus consecuencias favorables o adversas, en otras palabras, la permanencia de la ecuación "pensamiento = acción", mecanismo prelógico que se sustituye posteriormente por el pensamiento lógico y realista y que permanece reprimido en condiciones normales, actuando desde el inconciente y manifestándose en los sueños, los actos fallidos y los síntomas neuróticos y psicóticos.

Esta fase de pensamiento mágico es especialmente intensa durante el periodo que Freud [6] denominó "fase oral sádica de la libido" y determina fantasías agresivas de incorporación y destrucción del objeto amado asł como las ansiedades, persecutorias de la venganza fantaseada del objeto destruido hacia el sujeto (muerte y aniquilación), que Melanie Klein ${ }_{2}[7]$ describe magistralmente en su concepto de "posición eśquizo-paranoide" y que fueron citadas tangencialmente en la explicación anterior del miedo de engolfamiento y pérdida de ląidentidad y los límites correspondientes a la contribución de Margaret Mahler [8] sobre là fijación en la fase simbiótica del desarrollo humano. Con estas tres aproximaciones teóricas fragmentarias de Freud, Klein y Mahler, intentaré brevemente el cuadro clínico del paciente, sin pretender acotar todos los detalles y puentes lógicos del caso.

La homosexualidad pasiva del paciente se explica como su intento por recuperar su identidad masculina puesta en peligro por su depauperada relación temprana con su madre, quiẹ no proporcionó al paciente los medios para superar la fase de identificación primaria femenina. Éste introyectó la figura femenina, "la devoró", en términos simbólicos, en su fantasía como un intento compensatorio por mitigar la gran angustia y agresividad desencadenadas por sus frustraciones orales. La madre lo lactó 1 mes, y casi desde su 
nacimiento lo depositó con gran frecuencia en casa de su abuela, donde no podía recibir las atenciones y cuidados propios de un lactante. Posteriormente se encuentra con un padre agresivo y ausente, que impide una identificación masculina con él y que repite el mismo modelo frustrador y aniquilante que vivió con su madre en las épocas más tempranas. Pór consiguiente transfiere hacia él el mismo motante dé agresividad y temor que originalmente había depositado en su madre, y däda la persistencia de los mecanismos de pensamiento mágico omnipotente, fantasea la venganzà destructora del padre como consecuencia de las fantasías agresivas del paciente proyectadas én el padre (posición esquizo-paranoide).

Sustituye la figura del padre por individuos del mismo sexo y establece relaciones homosexuales pasivas con ellos désdè los '8 años," como un intento de mitigar la angustia de càstración incrementada por su posición esquizoparanoide, adoptando el " papel femenino frente al padre, en virtud de que éste quería a su esposa e hijas (identificación femenina secundaria). Su identificación femenina secundaria se vio estimulada y reforzada por la conducta estimulante de la madre y de las hermanas de adoptar roles femeninos (vestirse de mujer $y^{\prime \prime}$ jugar con'muñecas), ast como poir las agresiones de sus hermanos varones. $\mathrm{Si}$ a lo anterior agregamos que fue sometido a una intervención quirúrgica sobre sus genitales en pleno período edípico; no resulta sorprendente que el paciente haya incrementado sus fantasías de cástración y que no haya encontrado otra alternativa que la identificación femenina secundaria como único medio de poder sobrevivir a sus fantasías de aniquilación $\mathrm{y}$ destrucción, producto de su identificación femenina primaria, disolución simbiótica, fase oral sádica frustrada y posición esquizo-paranoide persistente.

La ambivalencia de la posición esquizo-paranoide provoca una culpa persecutoria del objeto destruido por la fantasía agresiva proyectada en él, "que es"vivida como amenaza de ániquilación. "Si "recordamos que el padre amenzó al paciente con matarlo si se volvía homosexual, podemos sospechar que para el pacienté, cada acto homosexual iba acompañado de una amenaza de muerte implícita proveniente de su deseo de destruir al padre, resultante de la transferencia más primitiva de destrucción a là madre en el proceso de identificación primaría femenina ỳ fase oral sádicá. Esto confirma ta persistencia de los mecanismós primitivos arcáicos presentes en cada acto homosexual. La búsqueda atropellante de parejas hómošexuales la realizó el paciente en el cábaret estafando individuos" y realizando sus " prácticas 'homosexuales diarias con varios individuos.

La consolidación de su identificación femenina secündaria se observa en su trabajo como secretario de una artista y en transvestismo. Desde luego, debajo de ella persiste su idèntificación fẻmenina primaria.

Si recordamós, el padre del paciente fallece en mayo de $1976^{\circ}$ y el paciente agudiza su cuadro clínico en mayo de $1977^{*}$ al aniversario de la muerte del 
padre. El paciente no asístió a su entierro, por lo que la cercanía dè la fecha de aniversario destapó su culpa persecutoria inconsciente producida por su deseo mágico de que muriera su padre. Para el paciente, su padre murió como consecuencia de su deseo fantaseado (pensamiento mágico omnipotente). Esta culpa persecutoria desencadenó en el paciente una cantidad de ansiedad superior a la que su aparato yoico podía tolerar, ya que el haber asesinado al padre en la fantasía significaba varias consecuencias: la venganza del pàdre contra el paciente, la abolición de la única figura que le permitía recuperar su identidad sexual, la pérdida de la posibilidad de protegerse de la desintegración del yo que significaba el enfrentamiento con las ansiedades de su identificación femenina primaria, la pérdida de una defensa contra sus fantasías orales sádicas directas hacia su madre, y la realización simbólica de la pérdida de su identidad personal. El resultado de estas ansiedades es la muerte. De ahí su intento suicida. Como si el paciente dijera: "por mi culpa murió aquél que me protegía de la destrucción de mi madre, no me queda otra alternativa que el suicidio". Lo que confirma el postulado psicoanalítico de que la homosexualidad es una defensa contra la desintegración de las fantasías orales sádicas, y la disolución simbiótica. El aniversario de la muerte del padre desencadenó el duelo patológico reprimido por el paciente durante un año y abatió sú homosexualidad defensiva originando el surgimiento de los mecanismos primitivos subyacentes. La actualización de éstos conduce irremisiblemente al suicidio.

Por último, la depresión y las tendencias paranoides se explican a través de distintos aspectos del mismo modelo. La depresión es la manifestación de la tristeza ocasionada por el rechazo del padre, los hermanos y los amigos por su conducta homosexual. Sin embargo, este rechazo tiene sus raíces en el rechazo original de la madre durante la fase oral, cuando el paciente fue descuidado y abandonado por 'ella, y debemos entender su depresión como una elaboración parcial de sus fantasías orales sádicas, de su fase simbiótica no superada y de su posición esquizo-paranoide. La depresión lo salvó de la psicosis, ya que el esquizofrénico es incapaz de elaborar siquiera en parte la frustración primaria de los mencionados períodos críticos del desarrollo humano.

La depresión es producto de un sentimiento de culpabilidad inconsciente ocasionado por las fantasías agresivas del paciente hacia sus objetos amados y necesitados. Sin embargo, esta depresión no puede ser elaborada completamente debido a la persistencia de las fantasias persecutorias que la realidad constantemente le alimenta, por esta razón, sù depresión se vuelve crónica, y periódicamente es negada mediante mecanismos de defensa maniacos que ciclicamente pierden su operatividad y su fuerza ante las ansiedades provenientes de la posición esquizo-paranoide no resuelta.

Por último, las tendencias paranoides, su fantasía de que todo el mundo lo busca para perjudicarlo, proviene de las fantasías originarias agresivas ha- 
cia sus objetos, los cuales van a devolver esta destructividad en forma retaliatoria (vengativa), mecanismo característico de la posición esquizo-paranoide en donde interviene la proyección de la propia agresividad y el pensamiento mágico omnipotente; estas fantasías se vieron confirmadas a lo largo de la vida del sujeto cuando su padre y sus hermanos lo agredieron incesantemente por homosexualidad, y posteriormente, en las reacciones de rechazo que el paciente mismo genera en las gentes que lo rodean al exhibir en forma manifiesta su personalidad homosexual.

En suma, lo que nos permite comprender el cuadro clínico del paciente en función de su historia personal y familiar es el modelo explicativo basado en el concepto de fijación pregenital en la etapa simbiótica, en la fase oral sádica o en la posición esquizo-paranoide, así como en los mecanismos defensivos empleados para mitigar la ansiedad proveniente de esta fijación: la identificación femenina secundaria, al culpa persecutoria suicida, su negación maniaca y la proyección de su propia agresividad en el afuera, que sintió en el allá y el entonces.

Para comprender las relaciones que se dan entre el nivel teórico y el observable voy a poner unos ejemplos extrafdos del caso anterior. Los conceptos 'de menor nivel de abstracción son aquellos que describen el cuadro clínico del paciente: conducta homosexual, vestirse con ropas de mujer, intento suicida, etc., cuya referencia puede ser dilucidada en función del comportamiento observable del paciente, su comunicación verbal y preverbal (gestos, mímica, movimientos corporales, etc.). Desde luego, esta referencia puede ser acotada a través de varias teorías semánticas que no serán discutidas en este trabajo y encierra una problemática en sí de complejidad extrema. Lo único que deseo señalar es que los conceptos que se refieren a 'las conductas observables del paciente son los que sirven de sustento a todo el aparato teórico psicoanalítico. A continuación, interviene un grupo de conceptós referentes a las emociones del paciente: angustia, odio, miedo, amor, culpa, etc., los cuales se relacionan con el material observable mediante la inferencia por analogía y la comunicación verbal, el paciente refiere verbalmente su angustia y el terapeuta la infiere a partir de las manifestaciones conductuales de la angustia y del conocimiento previo del uso de las palabras-que designan emociones. Este aspecto encierra toda la problemática de los conceptos de emoción, que no será,discutida aquí pero que se presupone en la teoría psicoanalítica.

También intervienen conceptos de creencias que acotan las diferentes expectativas del paciente ante los estímulos medioambientales: "el paciente cree que todo el mundo va a dañarlo", "cree que siendo mujer sería mejor aceptado", "cree que las "mujeres son más peligrosas que los hombres" etc., y que se relacionan con el material observable a través de cualquier teoría del aprendizaje que dé razón de las expectativas del paciente, por ejemplo, la repetición de una misma secuencia de estímulo-respuesta. . 
.. Luego, intervienen conceptos que establecen relaciones entre emociones, creencias y conductas, estos son los mecanismos de defensa: proyección, desplazamiento, negación, etc., y que se refieren a eventos mentales que pretenden explicar la conducta. Por ejemplo, “el paciente siente una intensa culpa por el rencor que guarda a su padre, y cree que este rencor es tan destructivo que mágicamente asesinó a su padre, por lo que espera que su padre se vengue de él, cree que es culpable de la pérdida de su objeto de salvación y que como castigo merece la muerte, esto "le ocasiona una angustia intolerable que lo conduce a su intento suicida". Los eventos mentales postulan hechos relacionales hịpotéticos analizables en términos de creencias, emoción y conducta; términos a su vez analizables en hechos observacionales. Sin embargo, la teoría psicoanalítica otorga nombres propios a una serie de eventos mentales conceptualizados como "conflictos psíquicos" y los clasifica en una gran variedad: mecanismos defensivos, impulsos sexuales y ágresivos, proyección, etc. Estamos en un nivel conceptual plenámente teórico y abstracto que requiere de varios puentes 'de comunicación con el material observable.

- Existe además una serie de hipótesis y teorías sobre el tipo de eventos mentales que ocurren durante las distintas fases del desarrollo humano y que pertenecen à la terminología más técnica psicoanalítica como: fase oral, fase simbiótica, posición esquizo-paranoide, que aglutinan conjuntos de eventos mentales relacionados mediante modelos conceptuales teóricos más abstractos aún correspondientes ar los -postulados más generales de la teoría psicoanalitica: el ; concepto de fuerzas psíquicas, energía psíquica, estructura de fuerzas, leyes del funcionamiento de las estructuras, nivel consciente e inconsciente, proceso primario y secundario, mecanismo-adaptativo, etc. Así por ejemplo; la explicación del intento suicida" del paciente puede darse en los siguientes términos: el paciente obró mediante una conducta sobredeterminada por los siguientes sistemas de fuerzas en conflicto: la persecución de sus objetos malos introyectados en su super-yo, desencadenada por la proyećción e introyección de su energía agresiva incrementada por las afrustraciones tempranas de su fase oral-sádica, mantenida por la permanencia de su fantasía omnipotente homicida inconsciente correspondiente a su preponderancia del proceso primario, lo que implica la fantasía vivida como culpa persecutoria, esto conforma la posición esquizo-paranoide, la cual desencadena una reactivación de la ansiedad de muerte producto de una fase simbiótica no resuelta por la fijación de la libido y la agresividad a la fase oral sádica, esta ansiedad de muerte sólo puede ser mitigada mediante la aniquilación del objeto malo introyectado y conduce al suicidio como consecuencia de la ley del principio económico de la reducción de la energía psíquica, cuya acumulación excesiva es vivida como dolor"intolerable, esto pone en crisis la propia identidad y amenaza la integridad de los límités dè yó, situáción que reactiva las pulsiones de muerte y la búsqueda de una situación libre de toda tensión, situa- 
ción equiparable en la fantasía a la muerte. Por consiguiente; el paciente intentó suicidarse.

Como he pretendido demostrar, los conceptos téricos del psicóanálisis pueden ser analizados en términos de conjuntos de relaciones de eventos mentáles, los cuales, pueden ser analizados en términos de eventos mentales. Éstos a su vez, pueden descomponerse en hechos relacionales entre emociones, creencias y conductas. Las emociones pueden analizárse en términos de comúnicación verbal e inferencia por analogía y las creencias, a través de modelos de aprendizaje de secuencias de patrones de estímulo-respuesta. Por último, están los conceptos de más bajo nivel que sè refieren a lás conductas y comú nicaciones verbales y preverbales del paciente; situaciones pertenecientes al material observable sobre el que descansan todas las conștrucciones teóricas del psicoanálisis.

Cada paso del análisis de las relaciones entre lo observable y lo teórico implica una problemática semántica presupuesta que ha sido desärollada por las diversas teorías del significado y la referencia, así como por la psicología filosófica que se ha ocupado de los problemas concernientes a los conceptos mentales, emociones, creencias y eventos. Mi intención no ha sido otra que esbozar un perfil de las relaciones entre los conceptos teóricos del psicoänálisis y los observacionales en términos distintos a la teoría mismas. No émpleé una aproximación formalista. Tampoco pretendo haber aclarado las relaciones entre lo téórico y lo observable: Sí; en cambio, "creo haber ofrecido una visión de la teoría psicoanalítica en isu funcionamiento aplicativo a un caso concreto, de la forma en ‘que-los conceptos teóricos se aplican ${ }^{\vee a}$ situaciones particulares observables, y sobre todo, que pueden ser analizados én términos de relaciones entre eventos mentales, ,emociones, creencias y conducta. Con esto se pueden percibir con $\iota$ más claridad las relaciónes existentes-entre la teoría psicoanalítica y la filosofía, así como las posibilidades que là semántica y la. filosofía de la mente abren a un estudio epistemológico del "psicoanálisis, complementario al que, la filosofía de la ciencia ha realizado en las últimas décadas. .

Miguel Kólteniuk

IŃstTTUTO dE INVESTIGACIONES FiLOSóficas

UntVerstdad Naciónal Aútónoma de México

1. Carnap, Rudolf: The Methodological Character of: Theoretical Concepts, en Minnesota Studies in the Philosophy of Science. Vol: I. University of Minnesota Press. U.S.A. 1962, pp. 38-76.

2. Moulines, Ulises-C. Reconstrucción Estructüral dè las teorias Fisicas. En prensa. 
3. Moulines, Ulises-C. "Un Concepto estructural de aproximación empírica", en Critica. Vol. VIII, No 24. México, diciembre 1976.

4. Kolteniuk, Miguel. "La naturaleza de los conceptos psicoanalíticos y su relación con la experiencia", en Diánoia. UNAM, 1975.

5. Socarides, W. Charles. "Homosexuality", en American Handbook of Psychiatry. Vol. III. Basic Books. New York, 1974, pp. 291-315.

6. Freud, S. Obras Completas. Ed. Biblioteca Nueva. Madrid, 1968.

7. Klein, Melanie. Obras completas. Paidos. Argentina, 1976.

8. Mahler, Margaret. Simbiosis Humana: las vicisitudes de la individuación. Joaquin Mortiz. México, 1972. 